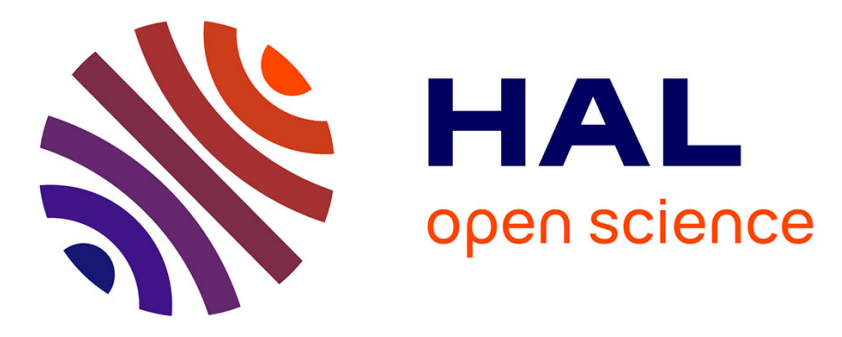

\title{
Affine kinematics in planar fibrous connective tissues: an experimental investigation
}

Charles Jayyosi, Jean-Sébastien Affagard, Guillaume Ducourthial, Christelle Bonod-Bidaud, Barbara Lynch, Stéphane Bancelin, Florence Ruggiero, Marie-Claire Schanne-Klein, Jean-Marc Allain, Karine Bruyère-Garnier, et al.

\section{To cite this version:}

Charles Jayyosi, Jean-Sébastien Affagard, Guillaume Ducourthial, Christelle Bonod-Bidaud, Barbara Lynch, et al.. Affine kinematics in planar fibrous connective tissues: an experimental investigation. Biomechanics and Modeling in Mechanobiology, 2017, pp.1-15. 10.1007/s10237-017-0899-1 . hal01529263

\section{HAL Id: hal-01529263 https://hal.science/hal-01529263}

Submitted on 14 Jun 2017

HAL is a multi-disciplinary open access archive for the deposit and dissemination of scientific research documents, whether they are published or not. The documents may come from teaching and research institutions in France or abroad, or from public or private research centers.
L'archive ouverte pluridisciplinaire HAL, est destinée au dépôt et à la diffusion de documents scientifiques de niveau recherche, publiés ou non, émanant des établissements d'enseignement et de recherche français ou étrangers, des laboratoires publics ou privés. 


\title{
Affine kinematics in planar fibrous connective tissues: an experimental investigation
}

\author{
C. Jayyosi ${ }^{1}$, J-S. Affagard ${ }^{2}$, G. Ducourthial ${ }^{3}$, C. Bonod-Bidaud ${ }^{4}$, B. Lynch ${ }^{2}$, S. Bancelin ${ }^{3}$, F. \\ Ruggiero $^{4}$, M-C. Schanne-Klein ${ }^{3}$, J-M. Allain ${ }^{2, \#}$, K. Bruyère-Garnier*,"\#, M. Coret ${ }^{5, \#}$ \\ ${ }^{1}$ Univ Lyon, Université Claude Bernard Lyon 1, IFSTTAR, UMR_T9406, LBMC, F69622, Lyon, France ; \\ ${ }^{2}$ LMS, Ecole Polytechnique, CNRS, Université Paris-Saclay, Palaiseau, France ; \\ ${ }^{3}$ LOB, Ecole Polytechnique, CNRS, INSERM, Université Paris-Saclay, Palaiseau, France ; \\ ${ }^{4}$ Institut de Génomique Fonctionnelle de Lyon, ENS-Lyon, CNRS, Université Lyon 1, Lyon, France ; \\ ${ }^{5}$ LUNAM Université, GEM, UMR CNRS 6183, Ecole Centrale de Nantes, Université de Nantes, France
}

Keywords: affine transformation; constitutive modeling; fibrous connective tissue; collagen fibers orientation; liver capsule; skin

\begin{abstract}
The affine transformation hypothesis is usually adopted in order to link the tissue scale with the fibers scale in structural constitutive models of fibrous tissues. Thanks to the recent advances in imaging techniques, such as multiphoton microscopy, the microstructural behavior and kinematics of fibrous tissues can now be monitored at different stretching within the same sample. Therefore the validity of the affine hypothesis can be investigated. In this paper, the fiber reorientation predicted by the affine assumption is compared to experimental data obtained during mechanical tests on skin and liver capsule coupled with microstructural imaging using multiphoton microscopy. The values of local strains and the collagen fibers orientation measured at increasing loading levels are used to compute a theoretical estimation of the affine reorientation of collagen fibers. The experimentally measured reorientation of collagen fibers during loading could not be successfully reproduced with this simple affine model. It suggests that other phenomena occur in the stretching process of planar fibrous connective tissues, which should be included in structural constitutive modeling approaches.
\end{abstract}

*Corresponding author: Karine Bruyère-Garnier, Phone: +33472142368, Email: karine.bruyere@ifsttar.fr

\# These authors contributed equally to the work. 


\section{Introduction}

As for most solids, the macroscopic mechanical behavior of soft biological tissues is deeply linked to their internal microstructural organization (Humphrey 2003; Gasser et al. 2006). For this reason, the development of analytical constitutive equations has been an active research area, in order to obtain structurally based models that predict the tissues behavior from information on their internal structures (Gasser et al. 2006).

Humphrey (Humphrey 2003) gives an extensive review of various biomechanical applications and contributions of constitutive models to the medical field. The development of surgical assistant robots is one of these examples: the behavior of the involved tissues and their injury thresholds have to be known and predicted accurately, to ensure a proper feedback (Kyriacou et al. 2002). These constitutive models can also help to optimize the rehabilitation procedures following injuries or surgeries, by suggesting the optimal loads that need to be applied to the tissues. For instance, Akeson et al. (Akeson et al. 1973; Akeson et al. 1980) showed that the immobilization of tendons or ligaments after injuries induces histological changes in the collagenous structure, degrading the mechanical properties of the tissues. Similarly, these models are also useful in the prediction of ruptured aneurysm risk, in order to design more efficient treatment schedules (Gasser 2011). They can also help to better understand surgical techniques (Holzapfel et al. 2002; Chauvet et al. 2010) and thus contribute to their upgrading.

These models also have a huge potential in the field of tissue engineering. Indeed, they offer a significant contribution to evaluate the injuries threshold of in-vivo tissues that the engineered tissue needs to reproduce, or to help ranking the structural properties which are important to reproduce (Butler et al. 2001). They may also help to estimate the mechanical signals experienced by the cells seeded in the scaffold (Obbink-Huizer et al. 2014), or to predict the mechanical consequences of the cell-induced remodeling (Loerakker et al. 2016).

The physiological basis of the constitutive models allows the straightforward incorporation of many different components and phenomena in the models. This is a major asset since these parameters are based on precise biological observations. It is then easy to model processes that directly influence the microstructure like ageing or pathologies, by degrading the parameters linked to the tissue structure or composition (Fung 1990). For instance, in the case of ageing, a microstructurally based model can take into account the elastin degradation that arises with ageing (Robert 2002).

Despite these numerous advantages, the development of such microstructural models presents significant challenges. One of the main concerns regards how the macroscopic deformation of the tissue is transmitted to the fiber network at the microscopic scale. Indeed, the way the transmission between these two scales is operated may have a great influence on the model response.

The affine assumption is used in most of the existing models. Within this hypothesis, the fibers are supposed to follow exactly the motion of the local volume in which they are embedded. Therefore, the fibers' stretching is equal to the tissue stretching in the particular direction of the fibers (Sacks 2003). Consequently, for a fiber family, the strain tensor of the fibers, $\underline{\underline{E}}_{f i b e r s}$, is given by:

$$
\underline{\underline{E}}_{\text {fibres }}=\boldsymbol{M}^{\boldsymbol{T}} \underline{\underline{E}} \boldsymbol{M}
$$

where $\underline{\underline{E}}$ is the Green-Lagrange strain tensor applied to the global tissue, and $\boldsymbol{M}$ the vector associated to the fiber family direction in the representative elementary volume.

The hypothesis behind the affine transformation assumption is that the structural phenomena arising from the interactions between fibers or between the fibers and the non fibrillar matrix are considered as not important for the tissue response. However, neglecting these phenomena may lead to an over- or 
under-estimate of the strain in the region of interest (ROI) since a part of the fibers displacement may come from interactions and constraints from the surrounding environment.

Experimental investigations of this fundamental assumption are only scarce (Billiar and Sacks 1997; Sacks 2003; Fan and Sacks 2014). This is due to the technical difficulties encountered for the observation of the fibrous microstructure during a mechanical assay, on the same sample and at the same location. Recent advances have been done through the combination of multiphoton microscopy (MPM) with mechanical assays (Screen and Evans 2009; Goulam Houssen et al. 2011; Keyes et al. 2013). MPM is a powerful technique to investigate the three-dimensional architecture of collagen-rich tissues since it offers intrinsic optical sectioning and deep penetration in the tissue. MPM modes of contrast include Two-Photon Excited Fluorescence (2PEF) and Second Harmonic Generation (SHG). 2PEF signal comes from elastin fibers and cells or from specifically stained components of the tissue (Deyl et al. 1980; Zoumi et al. 2002). Such specific staining can be used to determine the local strain field (Screen and Evans 2009; Mauri et al. 2013; Jayyosi et al. 2014). SHG specifically reveals the micrometer-scale organization of unstained collagen fibers (Zoumi et al. 2004; Raub et al. 2008; Goulam Houssen et al. 2011). Lately, SHG microscopy has been used in an increasing range of tissues to characterize the microstructural response to mechanical loading, such as skin (Bancelin et al. 2015), liver capsule (Jayyosi et al. 2016), aorta (Keyes et al. 2013), bone (Tang et al. 2015), cornea (Benoit et al. 2016), fetal membrane (Mauri et al. 2015), heart valve (Alavi et al. 2015), nerve (Vijayaraghavan et al. 2014) and tendon (Goulam Houssen et al. 2011).

In this study, the hypothesis of affine transformation is confronted to the experimental data from uniaxial and biaxial mechanical tests on planar connective tissues. Uniaxial tensile tests have been performed on skin (Bancelin et al., 2015), and elliptic bulge testing has been conducted on liver capsule (Jayyosi et al. 2016). Both experiments have been coupled with multiphoton microscopy imaging, to assess the kinematic of collagen fibers during the test. The local microscopic and macroscopic strains measured are then used to compute the theoretical evolution of the collagen fibers orientation, under the assumption of affine transformation. This theoretical orientation evolution is compared to the observed reorientation during these experimental assays, to assess the validity of the affine assumption.

\section{Material and Methods}

\section{Calculation of the theoretical reorientation of collagen fibers}

In the following, and when it is not mentioned, the mathematical formulation used is the one reported in Fan and Sacks (Fan and Sacks 2014). We restrict ourselves to planar deformations, since our data were obtained on biological membranes in which the fibers are mostly oriented within the same plane.

To test the affine model, we consider the evolution of collagen fibers orientation in the field of view of the multiphoton microscope during the mechanical test considered (whether uniaxial tensile tests or elliptic bulge tests). A theoretical reorientation under the affine assumption can be calculated based on the initial orientation of the fibers in the region of interest (ROI), and the strain applied to the tissue, as the affine model is purely kinematic.

Let us consider a family of fibers oriented in a direction $\boldsymbol{M}$ defined by an angle $\theta$ (see Fig. 1). Setting $\underline{\underline{F}}$ the 2D transformation gradient tensor, the new direction $\boldsymbol{m}$ of this fibers family associated to an angle $\beta$ after deformation reads:

$$
\boldsymbol{m}=\underline{\underline{F}} \boldsymbol{M}
$$


thus

$$
\beta=\tan ^{-1}\left(\frac{F_{21} \cos \theta+F_{22} \sin \theta}{F_{12} \sin \theta+F_{11} \cos \theta}\right)
$$

If we discard the non-diagonal component, we obtain the equation usually used in most reorientation models (Billiar and Sacks 1997; Chandran and Barocas 2005; Rezakhaniha et al. 2012):

$$
\tan \beta=\frac{\lambda_{y}}{\lambda_{x}} \tan \theta
$$

Let $\Gamma_{l}(\beta)$ be the fibers orientation distribution in the deformed state and $\Gamma_{0}(\theta)$, the orientation distribution in the reference state. As adjacent fibers remain adjacent, the number of fibers $d N$ around the angle $\theta$ is maintained after reorientation around the angle $\beta$ :

$$
\Gamma_{1}(\beta) d \beta=\Gamma_{0}(\theta) d \theta=d N
$$

therefore

$$
\Gamma_{1}(\beta)=\Gamma_{0}(\theta) \frac{d \theta}{d \beta}
$$

In the general case, the angular derivative is estimated by following the deformation of the surface element associated to a variation of angle $d \theta$ around $\theta$ into the same element for $\beta$, as done by Fan and Sacks (Fan and Sacks 2014). We then obtain, for a 2D transformation:

$$
\Gamma_{1}(\beta)=\Gamma_{0}(\theta) \frac{\boldsymbol{M}^{\boldsymbol{T}} \times \underline{\underline{\boldsymbol{C}} \times \boldsymbol{M}}}{\operatorname{det} \underline{\underline{\boldsymbol{F}}}}
$$

with $\underline{\underline{C}}$ the right Cauchy-Green dilation tensor calculated by $\underline{\underline{C}}=\underline{\underline{F}}^{T} \underline{\underline{F}}$.

In the case of a diagonal gradient of the transformation, we get the simple relationship:

$$
\Gamma_{1}(\beta)=\Gamma_{0}(\theta)\left(\frac{\lambda_{y}}{\lambda_{x}} \sin ^{2} \theta+\frac{\lambda_{x}}{\lambda_{y}} \cos ^{2} \theta\right)
$$

It is thus possible to compute the theoretical reorientation of each direction of the $2 \mathrm{D}$ space between $[0, \pi]$ knowing the kinematic of the transformation. The orientation distribution for the deformed configuration is inferred from the orientation distribution of the reference state, associated with the reoriented directions.

\section{Elliptic bulge test on liver capsule}

\subsection{Experimental set up}

The experimental data are based on the experimental work presented in Jayyosi et al. (Jayyosi et al. 2016). In this study, bulge tests on human liver capsule were performed under a multiphoton microscope to assess the changes that occurred in the fibrous structure during a biaxial loading. The liver capsule, or Glisson's capsule, is a fibrous connective membrane mainly made of type I collagen 
fibers and elastin fibers (Voss et al. 1980; Gelse et al. 2003). These two fibers networks are deeply intertwined and are considered to define the tissue response to a mechanical loading, which present a non-linear hyperelastic behavior.

Briefly, a customized air inflation device was developed to perform the bulge test under a two photon excitation microscope (NIKON, A1R MP PLUS ${ }^{\circledR}$ ) at the IVTV platform ("Ingénierie et Viellissement des Tissus Vivants", Engineering and Ageing of Living Tissues, ANR-10-EQPX-06-01). Inflation was conducted with pressure increments of 0.1 bar followed by pauses for imaging. SHG and 2PEF signals were then recorded on the entire thickness of the capsule samples. At the same time, the altitude of the apex was collected thanks to the displacement sensor of the microscope objective, to get an estimation of the macroscopic vertical displacement of the sample. In order to test different loading conditions, 3 configurations of the bulge test were developed: a circular one (referred to as R1), and 2 elliptic (referred to as R2 for the samples with a ratio 2 between the minor and major axis of the ellipse and $\mathrm{R} 4$ for a ratio 4). Therefore, some circular samples $(\mathrm{n}=7)$ have been loaded at the apex in an equibiaxial manner, and some elliptic samples ( $22 n=5$ and $R 4 n=5)$ have been subjected to nonequibiaxial loading.

\subsection{Data analysis and strain calculation}

The collagen fibers orientation distributions were determined on the obtained SHG images (see Fig. 2), at each loading step, using the OrientationJ plugin of ImageJ (Rezakhaniha et al. 2012) .

Macroscopic and microscopic measures of strain at the apex were also performed. The microscopic local strain fields at the apex were assessed using the method described previously (Jayyosi et al. 2014). Briefly, a grid of intrinsic markers was created by photobleaching at the apex of the capsule: a $5 \times 5$ grid of photobleached squares, as seen on figure 3, was made on an area of $260 \times 260 \mu \mathrm{m}^{2}$ in the field of view (which was $507 \times 507 \mu \mathrm{m}^{2}$ wide). The positions of these squared markers were collected at each loading step and used to compute local strain fields using the finite element method.

The macroscopic strain was estimated under the assumption of an ellipsoidal shape of the inflated capsule (Jayyosi 2015). The approximated ellipsoid dimensions were obtained from the apex vertical displacements, measured by the displacement of the microscope objective, and the size of the clamping ellipses. The stretch ratios in $\mathrm{X}$ and $\mathrm{Y}$ directions, $\lambda_{\mathrm{x}}$ and $\lambda_{\mathrm{y}}$ respectively, were then calculated from the ellipsoid meridian lengths in each direction. These meridian lengths were estimated by the second formula of Ramanujan (Eq. 9), that gives the approximation of an ellipse circumference (Ramanujan 1914):

$$
\begin{gathered}
L \approx \frac{\pi(a+b)}{4}\left[1+\frac{3 h}{10+\sqrt{4-3 h}}\right] \\
h=\left(\frac{a-b}{a+b}\right)^{2}
\end{gathered}
$$

where $a$ and $b$ are the semi minor and major axes of the ellipse. Thus, $a$ is the vertical displacement of the capsule apex and $b$ the dimension of the clamping ellipse in the X or Y direction. The stretch ratios $\lambda_{x}$ and $\lambda_{y}$ were then obtained by dividing this meridian length by the initial dimension of the clamping ellipse according to the case (R1, R2 or R4).

Consequently, since we calculated the strain at two different scales, the reorientation calculation was also conducted at two different levels. First the macroscopic strain calculated via the ellipsoidal assumption was used to compute the fibers reorientation on the whole images. The calculation was performed on the distribution of every image of the stack, at each pressure level, with the assumption of a homogenous strain across the thickness. Second, a more local approach was considered by focusing the analysis to the area of the photobleaching grid. Therefore a measure of the local orientation in each element of the mesh was made, in the mean plane of the grid. The theoretical fibers 
reorientation was calculated for each element of the mesh, based on the local strain calculated in that particular element.

\section{Uniaxial tensile test on skin}

\subsection{Experimental set up}

The used experimental data originate from Bancelin et al. (Bancelin et al., 2015), in which uniaxial tensile tests were performed under SHG microscope. Briefly, murine skins were harvested from the back of 4 week old wild-type mice; epidermis and hair were removed without altering the underlying dermis structure. These samples were cut in bone shape and were stretched in situ with a custom-made tensile device (see Fig. 4a). The stress/stretch curves exhibited a classical "J" shape: they had a toe region, a gradual increase (heel region) and a linear section, before rupture (see Fig. 4b). The microstructural organization was assessed by use of a custom-made multiphoton microscope. SHG images $\left(300 \times 300 \mu \mathrm{m}^{2}\right)$ showed fibrillar structures corresponding to collagen fibers, interrupted by round structures with no SHG signal corresponding to the hair follicles (see Fig. 4c). As the microstructural image acquisition was impossible during the stretching because of slight movements, loading was performed incrementally (see Fig. 4b), with a pause every 5\% of stretching.

\subsection{Data analysis and strain calculation}

In Bancelin et al.(Bancelin et al. 2015), the centers of hair follicles were tracked at each stretch level. They defined a Delaunay grid, which was used to compute the local stretch (see Fig. 5a-c). To reduce the effects of misplaced points, the mesoscopic strain was determined by averaging over the whole region of interest. Figure $5 \mathrm{~d}-\mathrm{f}$ show the components of the mesoscopic surface stretch tensor $\left(\lambda_{\mathrm{xx}}, \lambda_{\mathrm{yy}}\right.$, and the sliding angle $\omega$ ) as a function of the global stretch ratio $(\lambda)$. Along the traction direction, the $\lambda_{\mathrm{xx}}$ slope was equal to 1 , indicating a homogeneous deformation of the sample. Transversely to the traction direction, the $\lambda_{y y}$ response was found to be not linear. The initial increase was likely due to structural effects or water absorption; for higher stretch ratios, we observed the expected decrease. At the SHG scale, the sliding angle $\omega$ remained small $\left(<5^{\circ}\right)$, and thus was neglected to compute the theoretical fibers reorientation.

Independently from the strain analysis, the collagen fibers orientation distribution was extracted from SHG images at each step. A morphological filtering was used to create a map of the fibers orientation, and then a normalized histogram of the fibers orientation distribution was calculated (see Fig. $5 \mathrm{~g}$ ) on the field of view.

Using the mesoscopic strain, we computed the theoretical evolution of the normalized collagen fibrils distribution at each level of stretching.

\section{Results}

Experimental observations gave access independently to the strain and to the fibers orientation distribution at three different scales: 1) the orientation evolution on the whole image $\left(500 \mathrm{x} 500 \mu \mathrm{m}^{2}\right)$ as a function of the strain estimated from macroscopic motions for the liver capsules, 2) the orientation evolution on the whole image $\left(300 \times 300 \mu \mathrm{m}^{2}\right)$ as a function of the local measurement of the strain on the same region for the murine skin, and 3) the orientation evolution in 16 subparts of the image as a function of the local strain measured on the same subpart for liver capsule.

In each case, we computed the theoretical distribution of fibers orientation at each strain level using the affine assumption. This distribution is then compared to the measured one. 


\section{Maximal reorientation}

\subsection{Reorientation in the liver capsule for the whole image, based on macroscopic strain}

Figure 6 presents the measured initial distribution as well as the theoretical and experimental distributions at the highest pressure level for a human liver capsule. Each subfigure corresponds to a different type of biaxial loading. The examples were chosen so that they display a different initial orientation distribution. Although the analyses were performed on the full stack of images, the figures present only the evolution of a given optical plane of the liver capsule for the sake of clarity.

The main observation is that the affine assumption is not able to predict the reorientation observed experimentally. For the samples that showed an important reorientation during loading $\left(\sim 30^{\circ}\right.$, see Fig. $6 \mathrm{~b})$, the reorientation calculated from the macroscopic strain is clearly under estimated when compared to the experimental ones. In fact, the strain values measured in these experiments lead to a predicted reorientation of only few degrees. Therefore the reorientation model derived from the affine assumption can predict the fiber reorientation only for samples that present a limited reorientation $\left(<10^{\circ}\right.$, see Fig. $\left.6 \mathrm{c}\right)$.

For circular samples (R1 type), the hypothesis of an equibiaxial loading implied that the stretches were the same in all directions, so the $\lambda_{\mathrm{y}} / \lambda_{\mathrm{x}}$ ratio was equal to 1 . Therefore, the affine assumption predicted that no reorganization should occur $\left(\Gamma_{0}(\theta)=\Gamma_{l}(\beta)\right.$ with Eq. 8). However, a slight reorientation was observed experimentally.

\subsection{Reorientation in the mice skin for the whole image, based on local strain measured on the same region}

Figure 7 presents the measured initial distribution as well as the theoretical and experimental distributions at a high stretch level $(\lambda=1.5)$, close to the rupture stretch for two skin samples. The analysis was performed few micrometers below the surface to have the better signal/noise ratio, but similar results are obtained at the different imaging depths.

In both cases, the fibers are realigned in the direction of traction $\left(0^{\circ}\right)$. The initial distribution had more or less two peaks (around - and $+20^{\circ}$ ), while the experimental and theoretical final distributions had a single peak centered on the direction of traction. Despite this qualitative agreement, the affine assumption was not able to reproduce quantitatively the observed distribution, which was broader.

\subsection{Reorientation in the liver capsule for sub-regions of the photobleaching grid, based on local strain measured on the same region.}

Figure 8 presents the measured initial distributions as well as the theoretical and experimental distributions for each element of the photobleached grid mesh, for the same samples as in figure 6 . However, the strains used for reorientation calculation were determined locally here unlike figure 6 . The distributions of the fibers orientation were estimated on the same ROIs as the ones used for the strain determination. As the grid had $5 \times 5$ points, we had access to 16 sub-regions (which correspond to the 16 elements of the mesh). The positions of the sub-images reported in figure 8 correspond to the positions in the photobleached mesh.

Results are quite similar to those observed with the global orientation distribution. For the samples showing no reorientation (see Fig. 8a and c), the small difference between experimental and theoretical orientation distributions that was previously observed in figure $6 \mathrm{a}$ and $\mathrm{c}$ is not present anymore in the different elements of the mesh, or it is smaller than the noise. For the samples where the reorientation was important (see Fig. 8b), results are similar to those from the global orientation analysis, although the reorientation magnitude now depends on the location in the grid.

\section{Reorientation with strain}


The previous examples (see Fig. 6, 7 and 8) were obtained for the highest stretch reached before rupture, where we expected to observe the highest discrepancy between the model and the observations. We now perform similar comparison at intermediate loading steps.

\subsection{Liver capsule}

Regarding the liver capsule, figure 9 shows the reorientation observed in the sample R2 (same as in figures $6 \mathrm{~b}$ and $8 \mathrm{~b})$, but at a lower pressure level $(\mathrm{P}=0.2 \mathrm{bar})$ which corresponds to the beginning of inflation. Figure 9a corresponds to the global reorientation calculated from macroscopic strain (as in figure $6 \mathrm{~b}$ ) whereas figure $9 \mathrm{~b}$ presents the local reorientation in the photobleaching grid (as in figure $8 b)$.

Not surprisingly, the observed reorientation is lower than the one observed at higher pressure. However, an important gap between theoretical and experimental reorientations is still observed in some areas, for instance in quadrant $8\left(2^{\text {nd }}\right.$ row, $4^{\text {th }}$ column $)$ in the figure $9 \mathrm{~b}$. In quadrant $4\left(1^{\text {st }}\right.$ row, $4^{\text {th }}$ column), the beginning of the reorientation process can be observed with the disappearance of the initial dominant orientation into a rather isotropic area. This implies that the discrepancy between the affine assumption and the experimental observations is observed even at small loading values.

\subsection{Skin samples}

Regarding the skin, we have access to a larger number of samples, and to more intermediate stretch levels. Figures $10 \mathrm{a}$ and $\mathrm{b}$ present the same results as figure 7 but for a lower stretch level $(\lambda=1.2)$ corresponding to the end of the heel region of the stress-stretch curve. A slight difference between theoretical prediction and experimental data is observed in figure $10 \mathrm{a}$ - the observed orientation remaining very close to the initial one. On the other hand, no difference can be seen between the model and the observation in figure $10 \mathrm{~b}$, while the experimental orientation no longer corresponds to the initial one.

The same analysis was performed at each step of stretching, for 12 different samples (see Supplementary Figures 1, 2, 3, 4, 5). Not all samples have reached the highest stretch levels (for example, only 4 have reached $\lambda=1.5$ ): the other ones have broken earlier. We observed that the experimental orientation distributions were close to the initial ones in the heel region of the stressstretch curve (stress-stretch curves can be seen onSupplementary Figure 6 - the size of the heel region depends on the sample), and became more and more different in the linear region. Not so surprisingly, the predicted distributions were very close to the observed ones in the heel region, and became more and more different at increasing stretching in the linear region.

To obtain a more quantitative comparison, we used the Orientation Index (OI) scalar (Bancelin et al. 2015). The OI is obtained from the orientation distribution $\mathrm{I}(\theta)$ and the angle of the main orientation $\theta_{\max }$ :

$$
O I=\left[2 \frac{\int_{-90^{\circ}}^{90^{\circ}} I(\theta) \cos ^{2}\left(\theta-\theta_{\max }\right) d \theta}{\int_{-90^{\circ}}^{90^{\circ}} I(\theta) d \theta}-1\right] \cdot 100
$$

The OI is related to the fraction of fibers aligned with the direction of loading: for a single peak distribution, it is directly related to the width of the pic. It is therefore a useful quantity to analyze the reorientation for uniaxial loading. The figures $10 \mathrm{c}$ and $\mathrm{d}$ show the evolution of the experimental and predicted Orientation Index, and the difference between the two. We observed a double part behavior: at low stretches, we did not observe a significant difference between the experimental and predicted OIs; at higher stretches, the difference progressively increased. This behavior was found in almost all our 12 analyzed samples (see Supplementary Figure 7). 


\section{Discussion}

We have tested the validity of the affine assumption with respect to experimental observations in two planar fibrous tissues. The affine assumption is used in most multiscale models of connective tissues to relate the motions and stretches of the collagen fibers to the ones of the material volume embedding these fibers. Indeed, it is the simplest and most natural assumption since it supposes that the fibers follow exactly the kinematic of the representative elementary volume. Within this assumption, the knowledge of the transformation kinematics is sufficient to completely determine the kinematics of the collagen fibers.

We used multiphoton microscopy combined to mechanical assays to image the collagen fibers organization at different stretch levels. This was performed on two different tissues: the Glisson's capsule of human livers and the dermis of murine skins, which were subjected to respectively biaxial and uniaxial tensile tests. The multiphoton microscopy and more specifically the SHG contrast provided volume images of the collagen fibers with a micrometer resolution. We used these images to extract the fibers orientation in a ROI (the whole image or subparts). Therefore, we had access to a series of collagen fibers orientation distributions at increasing stretch levels.

The approaches we used were efficient to determine the planar orientation of the fibers. Effects of curvature (for liver capsule) or out-of-plane inclination of the fibers were not taken into account. Indeed, at the scale of the whole image, we did not observe significant bending of the liver samples, even at high load. The out-of-plane inclination of the fibers could exist, but is expected to be small in planar tissues. Moreover, we had no reason to assume that the reorientation would be more accurately predicted for out-of-plane motions than for in-plane motions, while the full 3D analysis would require a higher resolution in the $\mathrm{z}$-direction than the one used here. The same ROI was observed during the whole experiment. Still, as the tissue was stretched, it became thinner which implied that more and more fibers were observed in the same plane. As we did not observe any difference in the fibers orientation distribution along the thickness of the sample, and as we used normalized distribution, this effect was not likely to create a significant bias in our data.

Independently, we determined the stretch of the ROI, based on other information obtained from the microscope: displacement of the centers of the hair follicles for the skin samples, and displacement of the apex or of a photobleached grid in the fluorescence channel for the liver samples. The knowledge of the strain and of the collagen fibers orientation histogram on the reference image was sufficient, in the affine assumption, to determine the orientations of the fibers at each stretch level.

We had three approaches for the strain calculation, based on the specificity of each tissue. Clearly, the macroscopic measurement used on liver capsule was the less accurate, since it relied on strong assumptions, investigated in Jayyosi's thesis (Jayyosi 2015). First, the motion of the apex of the sample was determined by the vertical displacement of the focal plane. Comparison with stereocorrelation images showed an average error of $-0.35 \mathrm{~mm}$, leading to an underestimation of the strain down to $-5 \%$. This can lead to an underestimated reorientation. Second, the boundary conditions could create heterogeneous strains and induce an extrinsic non affine kinematic that would not come from structural phenomena. In fact, the slight reorientation of circular samples observed in figure 6a may come from heterogeneities in the radial strains induced by a non-uniform clamping, which would modify the equibiaxial loading into a non-equibiaxial tensile test. The difficult control of the boundary conditions was indeed highlighted during stereoscopic digital image correlation tests (Jayyosi 2015).

Local measurements seemed much more promising, although harder to perform. Two different methods were used, selected on the specificities of the tissues. For liver samples we photobleached a grid since this tissue exhibited an important fluorescence signal coming from the non-collagen matrix. Then, the displacement of the grid was tracked using classic DIC approaches and used to determine the strain in each of the 16 subparts of the grid. Skin samples did not show a fluorescence background, but exhibite well defined endogenous structures: the hair follicles. So, we tracked the center of each follicle, creating a grid on which we computed the strain. As the follicles were not uniformly 
distributed, and as their position tracking was not perfect, we averaged the computed strains on the whole image, which was less sensitive to measurement errors and provided parameters at the same scale for all samples.

We had thus access separately to an observed and a predicted histogram of fibers orientations at each stretch level. The direct comparison showed that the affine model did not predict correctly the evolution of the fibers orientation. Not surprisingly, it was at the largest stretches that we observed the greatest differences (see figures 6 and 7). At low stretch level (see figures 9 and 10), the model and the observations were in general very close, although important differences were observed in case of important reorientations (higher than $10^{\circ}$ ). Quantitative measurements on skin samples indicated that the differences between the predictions and the experimental observations appeared at the end of the heel region and increased in the linear region. These observations are consistent with the observation of two different responses of the tissue in the heel region and in the linear region (Bancelin et al. 2015).

This limitation of the affine transformation assumption has been previously reported in studies on other types of tissues, in particular in the study of Chandran and Barocas (Chandran and Barocas 2005). They compared two models of a tissue equivalent, a gel of collagen fibers embedded in a matrix. On one hand, they considered a model based on the affine assumption to predict the strain and reorientation in the tissue. On the other hand, they tested a network-based model that included information on the connection and force transmissions between fibers. The comparison of these two models led to significant differences in the model responses about the microstructural kinematic. Especially, the fibers orientations, predicted by the two modeling approaches, presented some important differences. The fibers orientation in the network based model seemed to be not correlated to the fibers strain. Therefore, these results indicated that the kinematic was not exclusively defined by the fibers strain. Thus, the authors highlighted the importance of identifying the relationship between the fibers network and the surrounding environment, to assess the role played by the fibers interconnections on kinematic. In particular, for tissues which force transmission to fibers is mainly done by other fibers, the affine assumption appears greatly limited, and the impact of the organization in an interconnected network cannot be discarded. On the opposite, if the force transmission comes mainly from the global loading conditions, then, fibers will more likely act independently and enforce the assumption of an affine kinematic.

The response of the fibers to the strain thus depends on the type of tissues. In certain types of tissue like pericardium, a good correlation was found between experimental measurements and simulation with the affine transformation model (Fan and Sacks 2014). In the study by Billiar and Sacks (Billiar and Sacks 1997), the simulations of biaxial tensile tests coupled with a reorientation model under the affine assumption allowed to get fibers reorientation very close to experimental observations made by SALS (Small Angle Light Scattering). In the present work, we studied two other types of tissues. It is noteworthy that the affine assumption overestimates the reorientation for skin samples, while it underestimates it for liver capsules.

The reorganization of the network seems to depend on other factors than only the applied strain to the individual fibers, at least for elementary volumes of few hundred of micrometer. The degrees of freedom of the fibers are also defined by the network structure and the links between fibers, which can increase or prevent the reorientation of a given fiber in a particular direction.

Therefore, the consideration of a reorientation model based solely on geometry seems quite limited to describe accurately the kinematic of fibers in planar fibrous tissues. Structural effects take indeed a huge part in the network reorganization, and the affine assumption presents important limitations when it does not take into account the interactions between fibers that influence the kinematic greatly. Affine assumption may be indeed relevant for small representative volumes, smaller than the fibers mesh size. However, at such small scale, the samples are unlikely to remain homogeneous in mechanical properties. At larger scales - as the ones we have been considering - the spatial organization of the fibers, and the stress transmission by the extracellular matrix, are likely to play 
significant roles on the fiber reorganization with stretch. Therefore, more complex assumptions including stresses transmission in the fibers mesh will provide more accurate results.

\section{Conclusion}

The affine transformation assumption that allows linking the fiber strain to the global transformation of the tissue seems to present important limitations. Using multiphoton microscopy imaging combined with mechanical assays, we measured independently the strain and the fibers orientations at different stretching. Results indicate that the fibers' kinematic is not entirely affine, but is over or underestimated depending on the tissue.

The structural phenomena induced by the interactions between fibers are then likely to be predominant in the deformation mechanisms of planar fibrous tissues. The affine assumption appears to be appropriate only in some specific cases, since it neglects the impact of the surrounding environment. Therefore, the development of constitutive models of planar fibrous tissues based on microstructure must include information about these interactions to predict faithfully the tissue behavior and link the macroscopic response to the microscopic organization.

\section{Disclosures}

None of the authors have any professional or financial conflict of interest.

\section{Acknowledgments}

The authors wish to thank Pr Mathias Brieu for useful discussions. This work was supported by the Programme Avenir Lyon Saint-Etienne (ANR-11-IDEX-0007) of Université de Lyon, within the program "Investissements d'Avenir" operated by the French National Research Agency (ANR), and by grants from Ecole Polytechnique (interdisciplinary project) and from Agence Nationale de la Recherche (ANR-13-BS09-0004-02 and ANR-10-INBS-04). 


\section{References}

Akeson WH, Amiel D, Woo SL (1980) Immobility effects on synovial joints the pathomechanics of joint contracture. Biorheology 17:95-110.

Akeson WH, Woo SL, Amiel D, et al (1973) The connective tissue response to immobility: biochemical changes in periarticular connective tissue of the immobilized rabbit knee. Clin Orthop Relat Res 356-62.

Alavi SH, Sinha A, Steward E, et al (2015) Load-dependent extracellular matrix organization in atrioventricular heart valves: differences and similarities. Am J Physiol Heart Circ Physiol 309:H276-84. doi: 10.1152/ajpheart.00164.2015

Bancelin S, Lynch B, Bonod-Bidaud C, et al (2015) Ex vivo multiscale quantitation of skin biomechanics in wild-type and genetically-modified mice using multiphoton microscopy. Sci Rep 5:17635. doi: 10.1038/srep17635

Benoit A, Latour G, Marie-Claire SK, Allain JM (2016) Simultaneous microstructural and mechanical characterization of human corneas at increasing pressure. J Mech Behav Biomed Mater 60:93105. doi: $10.1016 /$ j.jmbbm.2015.12.031

Billiar KL, Sacks MS (1997) A method to quantify the fiber kinematics of planar tissues under biaxial stretch. J Biomech 30:753-756. doi: 10.1016/S0021-9290(97)00019-5

Butler DL, Goldstein S a, Guilak F (2001) Functional tissue engineering: the role of biomechanics in articular cartilage repair. Clin Orthop Relat Res 122:S295-S305. doi: 10.1115/1.1318906

Chandran PL, Barocas VH (2005) Affine Versus Non-Affine Fibril Kinematics in Collagen Networks: Theoretical Studies of Network Behavior. J Biomech Eng 128:259. doi: 10.1115/1.2165699

Chauvet D, Carpentier A, Allain J-M, et al (2010) Histological and biomechanical study of dura mater applied to the technique of dura splitting decompression in Chiari type I malformation. Neurosurg Rev 33:287-94; discussion 295. doi: 10.1007/s10143-010-0261-x

Deyl Z, Macek K, Adam M, Vancíková O (1980) Studies on the chemical nature of elastin fluorescence. Biochim Biophys Acta 625:248-54.

Fan R, Sacks MS (2014) Simulation of planar soft tissues using a structural constitutive model: Finite element implementation and validation. J Biomech 47:2043-2054. doi:

10.1016/j.jbiomech.2014.03.014

Fung YC (1990) Biomechanics: Motion, Flow, Stress, and Growth. Springer-Verlag New York

Gasser TC (2011) An irreversible constitutive model for fibrous soft biological tissue: a 3-D microfiber approach with demonstrative application to abdominal aortic aneurysms. Acta Biomater 7:2457-66. doi: 10.1016/j.actbio.2011.02.015

Gasser TC, Ogden RW, Holzapfel G a (2006) Hyperelastic modelling of arterial layers with distributed collagen fibre orientations. J R Soc Interface R Soc 3:15-35. doi: 10.1098/rsif.2005.0073

Gelse K, Pöschl E, Aigner T (2003) Collagens - structure, function, and biosynthesis. Adv Drug Deliv Rev 55:1531-1546. doi: 10.1016/j.addr.2003.08.002 
Goulam Houssen Y, Gusachenko I, Schanne-Klein M-C, Allain J-M (2011) Monitoring micrometerscale collagen organization in rat-tail tendon upon mechanical strain using second harmonic microscopy. J Biomech 44:2047-2052. doi: 10.1016/j.jbiomech.2011.05.009

Holzapfel GA, Stadler M, Schulze-Bauer CAJ (2002) A Layer-Specific Three-Dimensional Model for the Simulation of Balloon Angioplasty using Magnetic Resonance Imaging and Mechanical Testing. Ann Biomed Eng 30:753-767. doi: 10.1114/1.1492812

Humphrey JD (2003) Review Paper: Continuum biomechanics of soft biological tissues. Proc R Soc A Math Phys Eng Sci 459:3-46. doi: 10.1098/rspa.2002.1060

Jayyosi C (2015) Caractérisation mécanique et microstructurale du comportement à rupture de la capsule de Glisson pour la prédiction du risque de lésions des tissus hépatiques humains. Dissertation. Université Claude Bernard Lyon 1

Jayyosi C, Coret M, Bruyère-Garnier K (2016) Characterizing liver capsule microstructure via in situ bulge test coupled with multiphoton imaging. J Mech Behav Biomed Mater 54:229-243. doi: 10.1016/j.jmbbm.2015.09.031

Jayyosi C, Fargier G, Coret M, Bruyère-Garnier K (2014) Photobleaching as a tool to measure the local strain field in fibrous membranes of connective tissues. Acta Biomater 10:2591-601. doi: 10.1016/j.actbio.2014.02.031

Keyes JT, Lockwood DR, Simon BR, Vande Geest JP (2013) Deformationally dependent fluid transport properties of porcine coronary arteries based on location in the coronary vasculature. $\mathrm{J}$ Mech Behav Biomed Mater 17:296-306. doi: 10.1016/j.jmbbm.2012.10.002

Kyriacou SK, Mohamed A, Miller K, Neff S (2002) Brain mechanics For neurosurgery: modeling issues. Biomech Model Mechanobiol 1:151-64. doi: 10.1007/s10237-002-0013-0

Loerakker S, Ristori T, Baaijens FPT (2016) A computational analysis of cell-mediated compaction and collagen remodeling in tissue-engineered heart valves. J Mech Behav Biomed Mater 58:173187. doi: 10.1016/j.jmbbm.2015.10.001

Mauri a., Perrini M, Mateos JM, et al (2013) Second harmonic generation microscopy of fetal membranes under deformation: Normal and altered morphology. Placenta 34:1020-1026. doi: 10.1016/j.placenta.2013.09.002

Mauri A, Ehret AE, Perrini M, et al (2015) Deformation mechanisms of Human amnion: Quantitative studies based on second harmonic generation microscopy. J Biomech. doi: 10.1016/j.jbiomech.2015.01.045

Obbink-Huizer C, Oomens CWJ, Loerakker S, et al (2014) Computational model predicts cell orientation in response to a range of mechanical stimuli. Biomech Model Mechanobiol 13:227236. doi: 10.1007/s10237-013-0501-4

Ramanujan S (1914) Modular equations and approximations to pi. Q J Math 45:350-372.

Raub CB, Unruh J, Suresh V, et al (2008) Image Correlation Spectroscopy of Multiphoton Images Correlates with Collagen Mechanical Properties. Biophys J 94:2361-2373. doi: 10.1529/biophysj.107.120006

Rezakhaniha R, Agianniotis a, Schrauwen JTC, et al (2012) Experimental investigation of collagen waviness and orientation in the arterial adventitia using confocal laser scanning microscopy. Biomech Model Mechanobiol 11:461-73. doi: 10.1007/s10237-011-0325-z 
Robert L (2002) Elastin, past, present and future. Pathol Biol 50:503-511. doi: 10.1016/S03698114(02)00336-X

Sacks MS (2003) Incorporation of Experimentally-Derived Fiber Orientation into a Structural Constitutive Model for Planar Collagenous Tissues. J Biomech Eng 125:280. doi: $10.1115 / 1.1544508$

Screen HRC, Evans SL (2009) Measuring Strain Distributions in Tendon using Confocal Microscopy and Finite Elements. J Strain Anal Eng Des 44:327-335. doi: 10.1243/03093247JSA491

Tang T, Ebacher V, Cripton P, et al (2015) Shear deformation and fracture of human cortical bone. Bone 71:25-35. doi: 10.1016/j.bone.2014.10.001

Vijayaraghavan S, Huq R, Hausman MR (2014) Methods of peripheral nerve tissue preparation for second harmonic generation imaging of collagen fibers. Methods 66:246-255. doi: 10.1016/j.ymeth.2013.08.012

Voss B, Rauterberg J, Allam S, Pott G (1980) Distribution of collagen type I and type III and of two collagenous components of basement membranes in the human liver. Pathol Res Pract 170:50 60. doi: $10.1016 / \mathrm{S} 0344-0338(80) 80155-5$

Zoumi A, Lu X, Kassab GS, Tromberg BJ (2004) Imaging coronary artery microstructure using second-harmonic and two-photon fluorescence microscopy. Biophys J 87:2778-2786. doi: 10.1529/biophysj.104.042887

Zoumi A, Yeh A, Tromberg BJ (2002) Imaging cells and extracellular matrix in vivo by using secondharmonic generation and two-photon excited fluorescence. Proc Natl Acad Sci U S A 99:110149. doi: 10.1073/pnas.172368799 


\section{Figure legends}

Fig. 1 Reorientation of fiber with main direction $\mathbf{M}$ in a direction $\mathbf{m}$ after transformation $\underline{\underline{\mathbf{F}}}$

Fig. 2 Calculation of the orientation distribution of collagen fibers thank to the ImageJ plugin OrientationJ developed by Rezakhaniha et al. (Rezakhaniha et al. 2012). Top: map of collagen fibers with their detected orientation; Bottom: associated histogram of fibers orientation.

Fig. 3 Typical photobleaching grid made at the apex of a sample of human liver capsule observed with multiphoton microscopy at 0.1 bar. The photobleached squares, characterized by a local loss of fluorescence, are used as nodes of a mesh to compute local strain. Photobleached squares are $20 \mu \mathrm{m}$ x $20 \mu \mathrm{m}$ spaced out by $40 \mu \mathrm{m}$. Green color corresponds to the fluorescence channel that shows the elastin fibers, while magenta color corresponds to the Second Harmonic Generation (SHG) signal which reveals collagen fibers.

Fig. 4 a) In situ custom made tensile test device for skin sample stretching. b) SHG image of a murine dermis, revealing the hair follicles (black holes) and the collagen network (in green). c) Mechanical behavior of the skin under uniaxial tensile testing. The nominal stress versus stretch curve exhibits the four classical regions. The small relaxations are due to the pauses in stretching for image acquisition

Fig. 5 Analysis of the skin SHG image. (a-c) skin local strain on the traction axis $\left(\lambda_{\mathrm{x}}\right)$, transversally $\left(\lambda_{\mathrm{y}}\right)$ and sliding angle $(\omega)$, differentiated from the displacements of the hair follicles. (d-f) Average values of the surface stretch components $\left(\lambda_{\mathrm{xx}}\right.$ and $\left.\lambda_{\mathrm{yy}}\right)$ and the sliding angle $(\omega)$ in the SHG image as a function of the global stretch ratio $(\lambda)(\mathrm{g})$ Collagen fibers orientation, extracted from SHG image.

Fig. 6 Distribution of collagen fibers orientation for human liver capsule, at the end of loading (just before rupture), for a circular sample (a), an elliptic sample R2 (b) and an elliptic sample R4 (c). The images were taken in the median plane of the liver capsule. In each subfigure, the initial distribution of fibers is presented in yellow, the orientation distribution measured experimentally is indicated in blue, and the theoretical distribution calculated from the macroscopic strain values is shown in red

Fig. 7 Distribution of collagen fibers orientation for murine skin, at 50\% strain (just before rupture), for samples with a one peak (a) and a two-peaks (b) initial distributions. In each subfigure, the initial distribution of fibers is presented in blue, the orientation distribution measured experimentally is indicated in dashed black, and the theoretical distribution calculated from the averaged local strain is shown in dotted dash red

Fig. 8 Experimental (blue), theoretical (red) and initial (yellow) distributions of the collagen fibers in the liver capsule for (a) circular, (b) elliptic R2, and (c) elliptic R4 samples. Each quadrant of the graph corresponds to a different element of the finite element mesh made on the photobleaching grid. Reorientation calculation is based on local strain values. The samples are the same ones as in figure 6 , at the same pressure level (just before rupture)

Fig. 9 Experimental (blue), theoretical (red) and initial (yellow) orientation distributions of the collagen fibers in the liver capsule after the first increment of pressure i.e $\mathrm{P}=0.2 \mathrm{bar}$, a) calculated from the macroscopic strain values and $b$ ) in each element of the finite element mesh calculated from local strain value (each quadrant corresponds to a different element of the mesh). The sample presented here are the same samples as in figures 6.b and 7.b, which presented important reorientation

Fig. 10 Reorientation of the collagen fibers at low strain for murine skin samples. (a-b) Initial orientation distribution of fibers (blue), experimentally measured orientation distribution (dashed black), and theoretical distribution calculated from the averaged local strain (dotted dash red) for the same samples as the ones infigure 7, for a strain of 20\%. (c-d) Evolution of the OIs measured (dotted black) and calculated (dashed black), and their difference (measured minus calculated) (red), as a function of the applied strain 